\title{
Review on the Synthesis Methods of Nano- Tungsten Oxide Dihydrate Colloid
}

\author{
Khizar Mushtaq ${ }^{1}$, Pui May Chou ${ }^{1 *}$, and Chin Wei Lai $^{2}$ \\ ${ }^{1}$ School of Computer Science and Engineering, Faculty of Innovation and Technology, Taylor's \\ University Lakeside Campus, No. 1, Jalan Taylor's, 47500 Subang Jaya, Selangor Darul Ehsan, \\ Malaysia \\ ${ }^{2}$ Level 3, Block A, Nanotechnology \& Catalysis Research Centre (NANOCAT), Institute for \\ Advanced Studies (IAS), University of Malaya, 50603 Kuala Lumpur, Malaysia
}

\begin{abstract}
Tungsten being a transition element, forms oxide compounds of various oxidation states that enables it to form nanocolloids of tungsten oxide dihydrate. Multiple methods have been used in recent years to synthesize nano tungsten oxide dihydrate, including sol-gel synthesis, electrochemical deposition, hydrothermal synthesis and anodization. However, a universally accepted synthesis method for this material is not offered. The most appropriate method and its corresponding processing parameters for the synthesis of nano tungsten oxide dihydrate colloids were presented in the present study. The objective of the present study was to investigate the effect of processing parameters, i.e. applied voltage, temperature and anodizing duration on the particle size of nanocolloids. It is found that anodization is the easiest, efficient, and cost-effective method to synthesize the colloidal solution of nano tungsten oxide dihydrate. Conducting the synthesis at room temperature at a voltage of $50 \mathrm{~V}$ for 60 minutes yields the product with particle size of $40-60 \mathrm{~nm}$, which can be used in wide array of applications. This paper also highlights the research gaps for future work and gives recommendations to extend this study particularly for the industrial application of tungsten oxide.
\end{abstract}

\section{Introduction}

Research on the nanomaterials has accelerated rapidly in recent years due to their usage in wide-ranging applications. Advancement in the nanotechnology has greatly influenced the materials engineering as it offers opportunities to develop and improvise the applications by creating better materials [1]. To be specific, nanostructures of transition metal oxides have significantly interested the researchers to study the unique properties exhibited by them particularly because they are characterized by multiple oxidation states. This allows them to form stable metal compounds resulting in exciting potential properties in the arena of superconductivity, microelectronics, photonics, and photocatalysis [2]. Amongst the numerous transition metals, the oxides of Tungsten element have fascinated

\footnotetext{
* Corresponding author: PuiMay.Chou@taylors.edu.my
} 
the researchers particularly, Tungsten Oxide, which is already being used in numerous applications.

Tungsten oxide $\left(\mathrm{WO}_{3}\right)$ is a transition metal oxide semiconductor which has proven to be a promising compound for various applications such as gas sensors, smart windows, electrochromic devices and photocatalysis [3-4]. The unique properties of this material allow for its greater and potential industrial application which has made it very popular among the researchers worldwide. According to the Web of Science, there have been in total of 24,576 publications related to tungsten Oxide, when searched in all the databases. Figure 1 illustrates that the number of publications has increased significantly on Tungsten oxide in recent years which shows the interest of the industrialists in this material and its demand in the future.

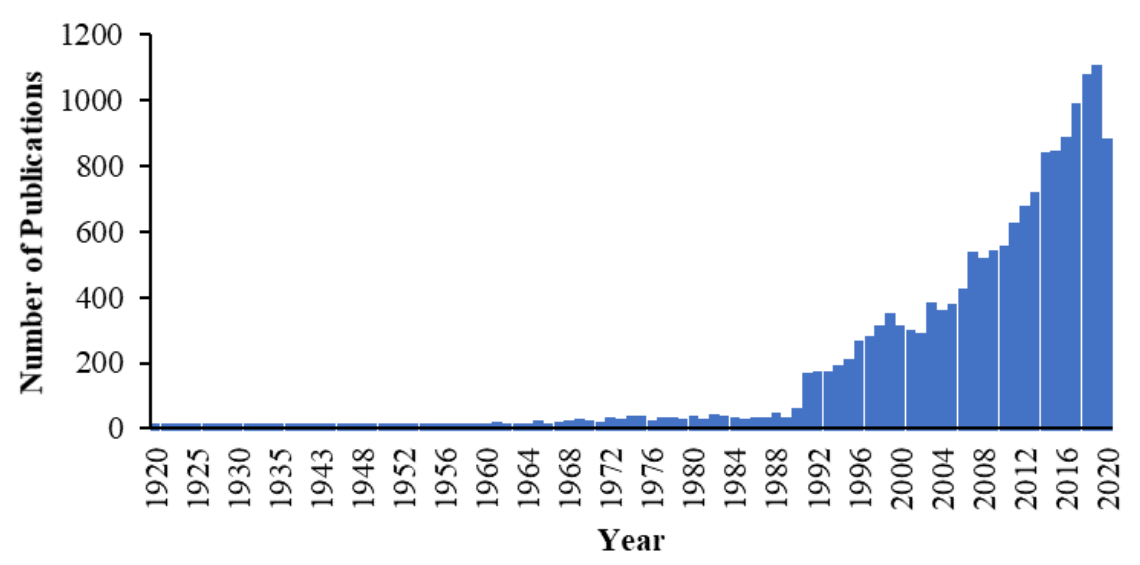

Fig. 1. Number of publications related to tungsten oxide per year as reported by Web of Science.

Tungsten oxide $\left(\mathrm{WO}_{3}\right)$ has a wide and tunable bandgap energy which ranges from 2.5 $2.8 \mathrm{eV}$, depending upon the type of the structure it possesses [5]. As Tungsten is a transition element and has many oxidation states i.e. 2,3,4,5 and 6, the metal oxides occur in various forms as well. Among the multiple forms of this compound, the nano structures of $\mathrm{WO}_{3}$, a polymorphous compound with trioxide, is the most commonly used form of tungsten oxide since it occurs due to the fully oxidized state of $\mathrm{W}^{6+}[6]$. Stable nanostructure of Tungsten trioxide possesses unique properties which provide enhanced surface area and high surfaceto-volume ratio for physical and chemical interactions, has greatly influenced the technologists to use this material in numerous applications as mentioned earlier.

The oxides of tungsten occur in various forms due to the variable oxidation states, the metal also forms tungsten oxide hydrates in many forms which can be labelled as $\mathrm{WO}_{3} \cdot n \mathrm{H}_{2} \mathrm{O}$, where $n$ is an integer [7-8]. The hydrates are usually formed between the ordinary hydrogen tungsten oxide hydrate, $\mathrm{H}_{2} \mathrm{WO}_{4}$, which is generally referred as tungstic acid, and the hydrogen tungsten oxide dihydrate $\mathrm{H}_{2} \mathrm{WO}_{4} \cdot \mathrm{H}_{2} \mathrm{O}$, where $n=1$ and 2 , respectively, for the above-mentioned hydrates. Other values of $n$ include $0.33,0.5,0.75$ and 1.8 which forms colloidal solutions for tungsten metal. Various $\mathrm{WO}_{3} \cdot n \mathrm{H}_{2} \mathrm{O}$ compounds are formed by corner sharing of octahedral structure that forms layers with the four $\mathrm{OH}^{-}$ groups lying in the $x y$ plane [9]. Concisely, Tungsten being a transition metal with multiple oxidation numbers, forms numerous oxides and hydrates with the valuable properties which makes it suitable to be used for various applications. Therefore, extensive studies on tungsten oxide nanostructures have been reported by other researchers [10-11]. 
Considering the significance of Tungsten Oxide Dihydrate due to its extensive properties, researchers have used multiple methods to synthesize this material. Until now, researchers have commonly used sol-gel Synthesis [11], hydrolysis [12], electrodeposition [13] and anodization [14] for the synthesis. Each of these methods have their own impact on the structure of the synthesized material including particle size, conductivity and antibacterial properties hence it is vital to study these methods for the uniformity in synthesis of nano structured tungsten oxide dihydrate for its wider usage in the applications [15-16].

This paper is aimed to review the impacts of various synthesis methods, which have been used in the past ten years, on the fabrication of nano tungsten oxide dihydrate colloids, as a comparative study of the synthesis methods has not been made prior to this research. This paper particularly focuses more on the Anodization method, considering the edge it has over other methods. Anodization being a simple and effective method, is a clean and safe synthesis method with no environmental setback [17-18]. The paper also investigated the effects of processing parameters used for anodization, on the formation of synthesized materials as the controlling parameters are not presented universally for the future research. By reviewing the methods, a generalized method for the synthesis is presented and suitable processing parameters to achieve the optimum properties are also recommended. The study will help the researchers to invest the time on the advancement and applications of nano Tungsten oxide dihydrate colloids instead of wasting time to work out an appropriate synthesis method. Future researchers will be able to save chemicals, resources, time and cost during their experimental work as they do not require to synthesize the material using different methods. This paper also aids the future researchers by highlighting the research gaps related to nano tungsten oxide dihydrate colloids thus promoting greater study on the material.

\section{Synthesis methods and their impacts on the formation of tungsten oxide nanostructures}

Properties of tungsten oxide including its morphology, crystallinity, stoichiometry and particle size are essential for its usage on potential applications thus researchers have laid great emphasis on synthesizing tungsten films to engineer the required chemical and physical properties of tungsten oxide. Researchers have exploited several liquid-phase synthetic approaches to tailor the nanostructures of tungsten oxide because of their greater control over the morphology as well as their implementation at relatively lower temperatures [19]. In this section, the most commonly used synthesis methods are comprehensively reviewed. Their impact on the formation of tungsten oxide nanostructures will also be discussed.

\subsection{Sol-gel synthesis}

Sol-gel synthesis is categorized as a wet-chemistry coating technology. This synthesis method is very vital for materials engineering and development, mainly due to its broad commercial applications as well as low production costs and large coating area capability. The principle of formation of metal oxide colloids using this technique particularly rely on polymerization reactions of molecular precursors [20].

Sol-gel synthesis method implicates a molecular precursor solution at the beginning of the reaction to form a network gel structure or discrete particles. Metal alkoxides dissolved in an appropriate organic solvent such as tungsten oxo-tetra-n-butoxide dissolved in ethanol and inorganic precursors (metal salts) dissolved in aqueous solutions, for instance, tungstic 
acid powder in hydrogen peroxide, are used as molecular precursors for the Sol-gel process. Precursors involves suitable gelation mechanism in various forms of hydrolysis and polycondensation subsequently allowing them to alter into solid oxide networks possessing unique properties in terms of morphology and porosity. After the formation of sols, film is deposited onto the desired substrates by dip-coating, spin-coating or spraying followed by drying and calcination processes to achieve the stoichiometric and crystalline nano-tungsten oxide films but in the case of hydrated $\mathrm{WO}_{3}$, post-annealing treatment is mandatory to be performed to obtain the crystallized form [10].

Interesting morphologies of tungsten oxide can be obtained using sol-gel synthesis method. In recent work of Chai et al. [11], nanocrystalline structure of tungsten oxide was obtained by controlling the mixing sequences during precursor preparation and aging process. In their experiment, $40 \mathrm{ml}, 0.15 \mathrm{M} \mathrm{Na}_{2} \mathrm{WO}_{4}$ was added drop by drop from $25 \mathrm{ml}$ burette into $15 \mathrm{ml} 3 \mathrm{Mol}$ of nitric acid under vigorous stirring and samples were left aging at $25{ }^{\circ} \mathrm{C}$ for 30 days [11]. The experimental parameters are summarized in Table 1 . According to their findings for the tungsten oxide formed by dropping sodium tungstate into nitric acid, no structure was detected after the first day of aging. The structural changes were only noticed after 5 days of aging which was further improved in terms of uniformity after $30^{\text {th }}$ day of aging. After the 30-day aging duration, nanoplatelets of 200-600 nm particle size were obtained by the authors [11].

In other work, microporous tungsten oxide thin films were prepared by Alsawafta et al. [21]. Films were prepared using polystyrene microsphere template and peroxitungstic acid precursor followed by introduction of gold nanoparticles. The scanning electron microscopy (SEM) image of nonporous tungsten oxide film showed the particle size of $100 \mathrm{~nm}$ was achieved. The study also compared the electrochromic properties of gold doped tungsten oxide film with standard nano tungsten oxide and it was found that gold doped tungsten oxide films had better electrochemical properties. The procedure used to conduct the study is shown in Figure 2 [21]. Conclusively, sol-gel is an effective method for the synthesis of nano tungsten oxide with an ability to tune the resulting material with unique and desired properties. However, the process has some drawbacks specifically the high cost of raw materials to produce sol, large shrinkage of the material during gelation process and drying of gels as well as long processing time to complete the procedure. Therefore, another synthesis method, which is hydrothermal synthesis is studied and described in the following sub-section.

\subsection{Hydrothermal synthesis}

Hydrothermal treatment is a simple and cost-effective technique that offers significant advantages in controlling the product morphology and size at high temperature and pressure with good homogeneity. Typically, the hydrothermal treatment begins with the preparation of a precursor solution such as $\mathrm{H}_{2} \mathrm{WO}_{4}, \mathrm{WCl}_{6}$ and $\mathrm{Na}_{2} \mathrm{WO}_{4}$ solution. The precursor is then added with appropriate chelating ligands or capping reagents and kept at an elevated temperature that ranges from $120-300{ }^{\circ} \mathrm{C}$ for a desired period, allowing the nucleation and growth of the crystallites. The apparatus consists of a steel vessel, known as autoclave, lined with PTFE or alloys that withstand corrosion and in which the liquid containing the precursors is fed. The autoclave is hermetically sealed and at the lower side, heating is applied resulting in a temperature gradient between the lower and the upper ends. Consequently, due to supersaturation of the solute in the cooler zone, nucleation, followed by precipitation, the growth of the compounds occurs [10]. 


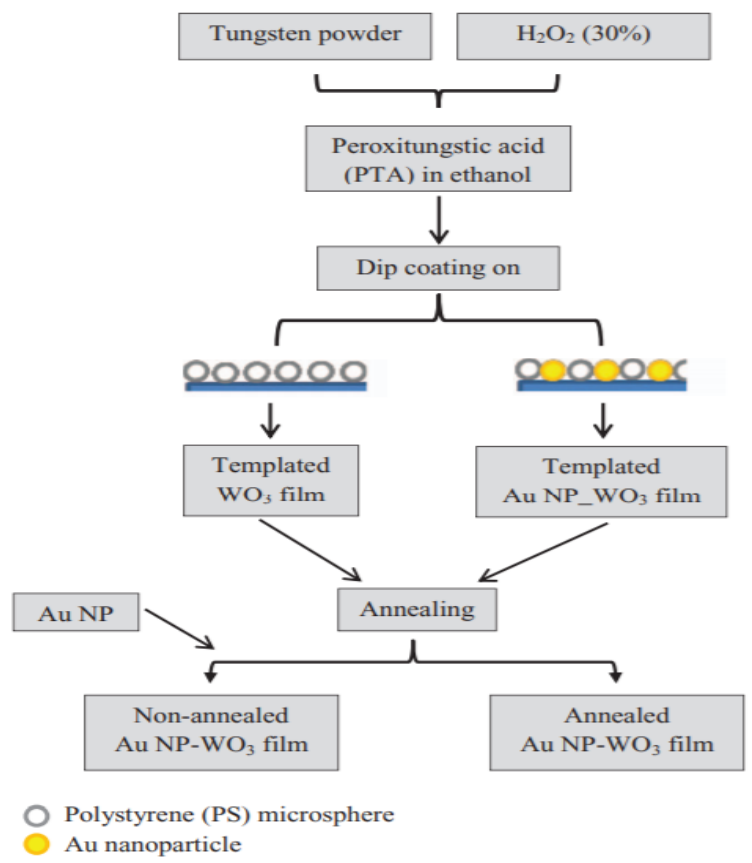

Fig. 2. Flow chart demonstrating the steps involved in fabrication of gold doped tungsten oxide films via sol-gel method [21].

Tehrani et al. [22] prepared $\mathrm{WO}_{3}$ nanostructures using hydrothermal technique at low cost, compatible with the environment and controllability of particle size, shape, and stoichiometry of the nanostructures. The author used 0.005 Mol Sodium tungstate $\left(\mathrm{Na}_{2} \mathrm{WO}_{4} .2 \mathrm{H}_{2} \mathrm{O}\right), 0.025 \mathrm{~mol}$ sodium sulfate $\left(\mathrm{Na}_{2} \mathrm{SO}_{4}\right)$ and $0.006 \mathrm{~mol}$ citric acid $\left(\mathrm{C}_{2} \mathrm{H}_{8} \mathrm{O}_{7} \cdot \mathrm{H}_{2} \mathrm{O}\right)$ to synthesize the nanostructures. For the experiment, sodium tungstate, citric acid and sodium sulfate were dissolved in $50 \mathrm{ml}$ deionized water. $2 \mathrm{M}$ Hydrochloric acid was gradually added into an aqueous solution when a transparent solution was formed. This was done to obtain the $\mathrm{pH}$ level of 2 . The solution was stirred for $10 \mathrm{~min}$ and transferred to a $100 \mathrm{ml}$ Teflon-lined autoclave. The hydrothermal synthesis was done for different durations of $6,12,24$, and $36 \mathrm{~h}$ at a fixed temperature of $180{ }^{\circ} \mathrm{C}$. The obtained precipitate was then filtered and washed thoroughly by deionized water and ethanol. The final product was put in an oven for $10 \mathrm{~h}$ at $60{ }^{\circ} \mathrm{C}$. After a long process, author obtained the product with maximum particle size of $73 \mathrm{~nm}$ after 24 hours duration of hydrothermal synthesis. It was also concluded that the tungsten oxide nanostructures can be used in optoelectronic devices as it was proved that synthesized samples were transparent in a wide range of visible light region [22].

Wang et al. [23] used hydrothermal synthesis method to fabricate tungsten oxide nanocube morphology. The study formed the nanocubes with aggregated size of $0.5-1 \mu \mathrm{m}$ as dozens of nanosheets with the thickness of $30-40 \mathrm{~nm}$ were stacked together to form tungsten oxide into nanocube morphology. As-prepared tungsten oxide nanocubes were compared with $\mathrm{WO}_{3} \cdot \mathrm{H}_{2} \mathrm{O}$ and $\mathrm{WO}_{3} \cdot 0.33 \mathrm{H}_{2} \mathrm{O}$ to conclude that tungsten oxide nanocubes possess higher photocatalytic performance as compared with the other two materials this suggesting the possible application of tungsten oxide in the area of wastewater purification [23]. The process of obtaining tungsten oxide is illustrated in Figure 3.

Hydrothermal synthesis is an effective method to produce metal oxides, but it has several disadvantages, as mentioned in Table 1 . The processes involved in this synthesis method are difficult to control which can lead to mishaps and add to the costs as well as 
wastage of resources if the process is mishandled. Moreover, the method requires expensive equipment such as autoclave to conduct the experiment.
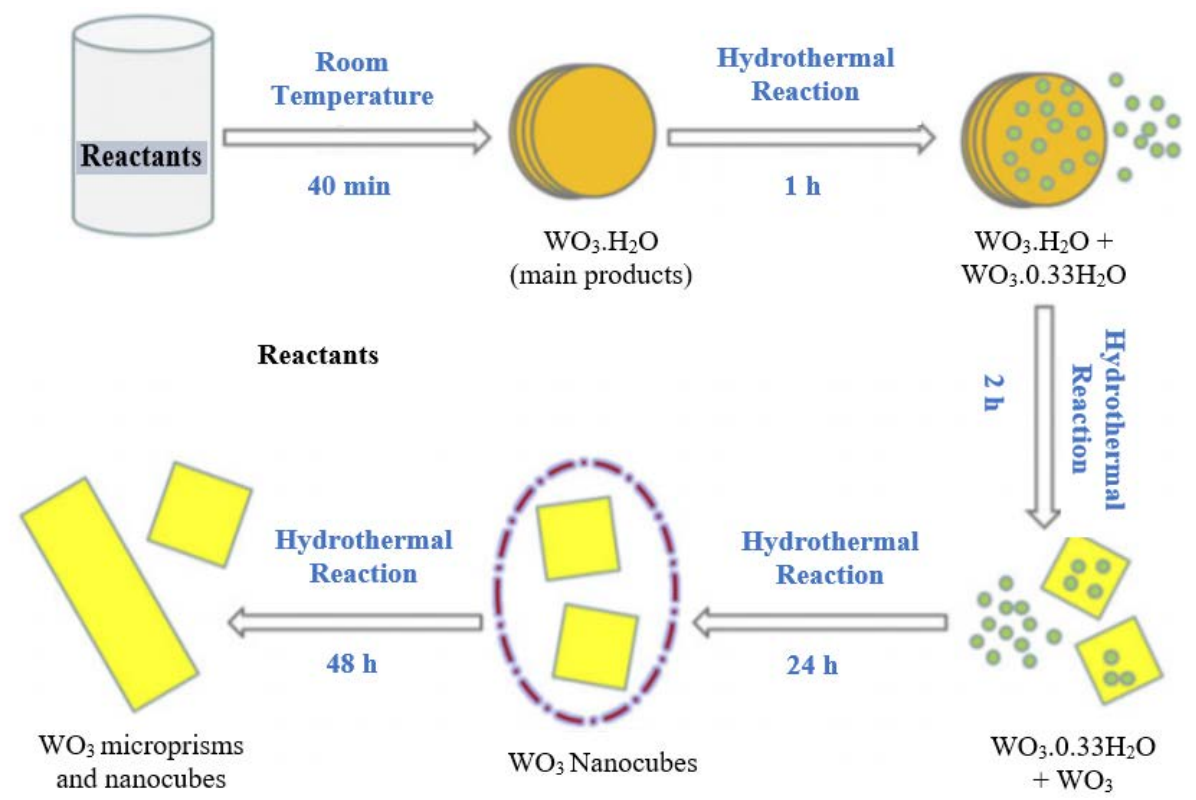

Fig. 3. Schematic illustration of the formation of tungsten oxide nanocubes via hydrothermal synthesis [23].

\subsection{Electrochemical deposition}

Electrochemical deposition is an attractive synthesis technique due to various benefits such as low cost, large area coating ability, and precise thickness control. In the electrodeposition process, an external potential is applied between a working electrode and counter electrode, with both immersed in an electrolyte solution. Metal oxide film is formed by the accumulation of metal ions present in the electrolyte solution. The process usually requires only a small applied voltage and the deposition duration ranges from 1 to 30 minutes as excessive applied voltages and prolonged reaction time will result in the formation of compact films with low porosity [10]. The morphological properties of the resulting $\mathrm{WO}_{3}$ can be controlled by an appropriate selection of the electrolyte solution and additives. The most popular choice of electrolyte employed is the peroxitungstic acid solution, while alcohol such as isopropanol or ethanol can be added to the solution to increase the stability [24].

Lin et al. [24] conducted the study by synthesizing tungsten trioxide thin films by electrodeposition on indium tin oxide glass to form $\mathrm{WO}_{3}$-coated glass. For the synthesis, the electrodeposition time and current were varied to have the greater control over the film thickness and morphology. The used tungsten metal powder and a low concentration $(34.5 \%-36.5 \%)$ of hydrogen peroxide $\left(\mathrm{H}_{2} \mathrm{O}_{2}\right)$ as primary materials used for the preparation of peroxitungstic acid. Dissolving $3.0 \mathrm{~g}$ of tungsten metal powder in $30 \mathrm{ml}$ of $\mathrm{H}_{2} \mathrm{O}_{2}$ yielded a colorless peroxitungstic acid solution. The study concluded that the thickness of tungsten thin films increased with the increase in electrodeposition time and current. The researcher achieved the films in the range of $132 \mathrm{~nm}$ to $216 \mathrm{~nm}$ after annealing process at $250^{\circ} \mathrm{C}, 500$ ${ }^{\circ} \mathrm{C}$, and $700{ }^{\circ} \mathrm{C}$. Figure 4 shows the experimental setup used by Lin et al. [24] for their studies. 
Table 1. Advantages and disadvantages of sol-gel synthesis and hydrothermal synthesis methods.

\begin{tabular}{|c|c|c|c|c|c|}
\hline $\begin{array}{l}\text { Synthesis } \\
\text { Method }\end{array}$ & Advantages & Disadvantages & $\begin{array}{c}\text { Experimental } \\
\text { Parameters }\end{array}$ & $\begin{array}{l}\text { Particle } \\
\text { Size } \\
(\mathbf{n m})\end{array}$ & References \\
\hline 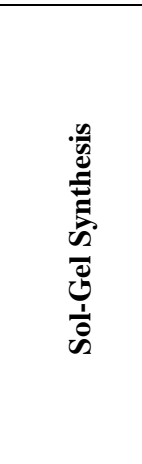 & $\begin{array}{ll}\text { - } & \text { Low } \\
\text { processing } \\
\text { cost } \\
\text { - } & \text { Controllable } \\
\text { film texture } \\
\text { and } \\
\text { structures }\end{array}$ & $\begin{array}{l}\text { Time consuming } \\
\text { due to long and } \\
\text { numerous } \\
\text { processes } \\
\text { - High cost of raw } \\
\text { material } \\
\text { - } \begin{array}{l}\text { Prescence of } \\
\text { large }\end{array} \\
\text { concentrations of } \\
\text { pores } \\
\text { - } \begin{array}{l}\text { Large volume } \\
\text { shrinkage }\end{array}\end{array}$ & $\begin{array}{l}\text { - } 40 \mathrm{ml} \text { of } 0.15 \\
\mathrm{M} \mathrm{Na}_{2} \mathrm{WO}_{4} \\
\text { was added into } \\
15 \mathrm{ml} 3 \mathrm{M} \\
\text { nitric acid } \\
\text { under stirring } \\
\text { - Samples were } \\
\text { left for aging } \\
\text { at } 25^{\circ} \mathrm{C} \text { for } 30 \\
\text { days }\end{array}$ & $200-600$ & {$[4,13,19]$} \\
\hline 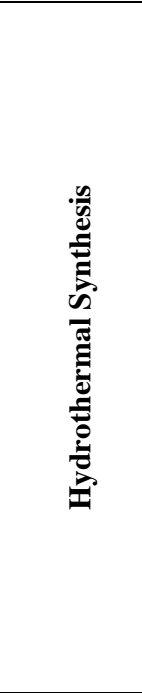 & $\begin{array}{l}\text { Ability to } \\
\text { create } \\
\text { crystals for } \\
\text { substances } \\
\text { which are } \\
\text { unstable at } \\
\text { their } \\
\text { melting } \\
\text { points }\end{array}$ & $\begin{array}{ll}\text { - } & \text { Processes are } \\
\text { difficult to } \\
\text { control } \\
\text { - } \\
\text { Expensive } \\
\text { autoclaves are } \\
\text { required }\end{array}$ & $\begin{array}{l}0.005 \mathrm{~mol} \\
\text { Sodium } \\
\text { tungstate, } \\
0.025 \mathrm{~mol} \\
\text { sodium } \\
\text { sulphate and } \\
0.006 \mathrm{~mol} \\
\text { citric acid } \\
\text { were dissolved } \\
\text { in } 50 \mathrm{ml} \\
\text { deionized } \\
\text { water } \\
2 \mathrm{M} \\
\text { Hydrochloric } \\
\text { acid was } \\
\text { added } \\
\text { Durations of } 6, \\
12,24, \text { and } 36 \\
\text { h at } 180^{\circ} \mathrm{C}\end{array}$ & $17-73$ & {$[19,22-23]$} \\
\hline
\end{tabular}

In other studies, More et al. [25] synthesized nano-granular tungsten oxide thin films using $100 \mathrm{ml}$ of 0.5 peroxitungstic solution by reacting with $9.192 \mathrm{~g}$ tungsten metal powder with $100 \mathrm{ml}$ of $30 \%$ hydrogen peroxide at room temperature. Author obtained the electrodeposited tungsten oxide films ranging from $30 \mathrm{~nm}$ to $60 \mathrm{~nm}$ size which were later used for electrochromic characterization. The films were found to have excellent coloration efficiency therefore it was recommended by the author that tungsten oxide thin films can potentially be used for smart window applications [25].

Although electrochemical deposition is one of the cheapest and simplest methods, it is difficult to ensure the uniformity of distribution of particles on the product or material to be produced. With lack of uniformity, it is difficult to produce the material with similar properties thus the results might differ if researchers used this method. This downside encouraged the author to study about electrochemical anodization for the synthesis of nano tungsten oxide dihydrate colloids.

\subsection{Anodization technique}

In a typical anodization setup, a working and a counter electrode are immersed in an electrolyte solution where a metal substance is used as a working electrode whereas 
platinum foil or carbon electrode is usually utilized for the counter electrode. The electrode where ions are oxidized or neutral substances lose electrons, is called anode whereas ions are reduced or neutral substances gain electrons on the surface of a cathode. The anodization setup is supported by applying the electric field between the two electrodes for a desired period and the electrical current will result in electrochemical reactions on the surface of both the electrodes, which leads to anodic corrosion through an oxidation process causing the materials to be released into the electrolyte medium from corrosive oxidation of the anode $[14,26]$.

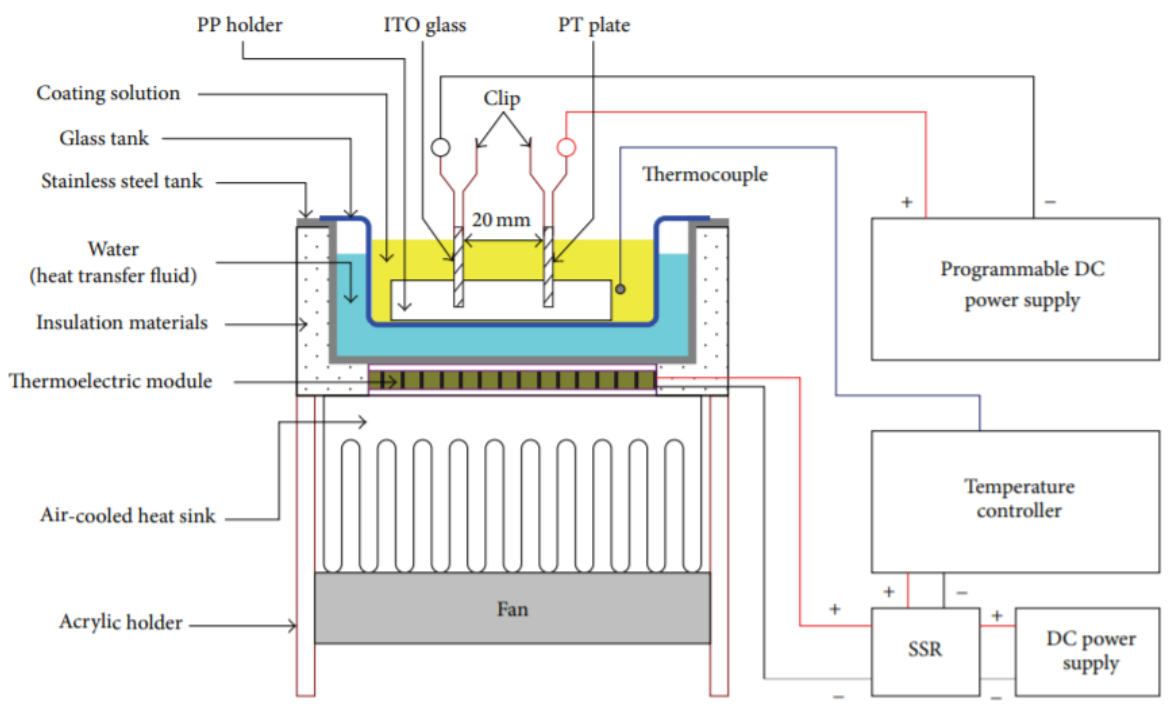

Fig. 4. Experimental setup used for the synthesis of tungsten trioxide thin films via electrochemical deposition method [24].

Extensive work has been carried out by the scholars in the past to synthesize nano tungsten oxide using anodization [27-28] but there is no universally accepted experimental setup. According to some researchers [7, 10], anodization of $\mathrm{WO}_{3}$ is similar to the anodization of $\mathrm{Al}_{2} \mathrm{O}_{3}$ while some emphasize the importance of using Fluoride ions for the formation of nano tungsten oxide.

Ranjbar et al. [26] fabricated nanoparticles of tungsten oxide dihydrate by anodizing tungsten rods in diluted $\mathrm{HCl}$. As a typical anodization setup, two tungsten rods were placed $1 \mathrm{~cm}$ parallel to each other into a $0.02 \mathrm{M} \mathrm{HCl}$ electrolyte followed by applying $60 \mathrm{~V}$ voltage between the two cathodes. Consequently, the anode surface began to corrode and was released gradually into the electrolyte hence forming the tungsten oxide dihydrate colloids of $5.1 \mathrm{~nm}$ particle size. Experimental Setup of anodization is shown in Figure 5.

Other researchers [29-30] conducted the studies on the synthesis of tungsten oxide by varying the controlling parameters used in anodization process. Effect of each of the anodizing parameters were found to have some impact on the structures of tungsten oxide thus this method can allow great structural changes which can further help the researchers to synthesize the material with desired properties. The effects of processing parameters are studied in detail in the following sections.

The methods described in this section are frequently used by the researchers over the years to synthesize the nanostructures of tungsten oxide but each of the synthesis techniques has their own drawbacks, which make it difficult for the researchers to produce 
the nanostructures with consistent properties. The advantages and disadvantages of electrochemical deposition and anodization methods are summarized in Table 2.

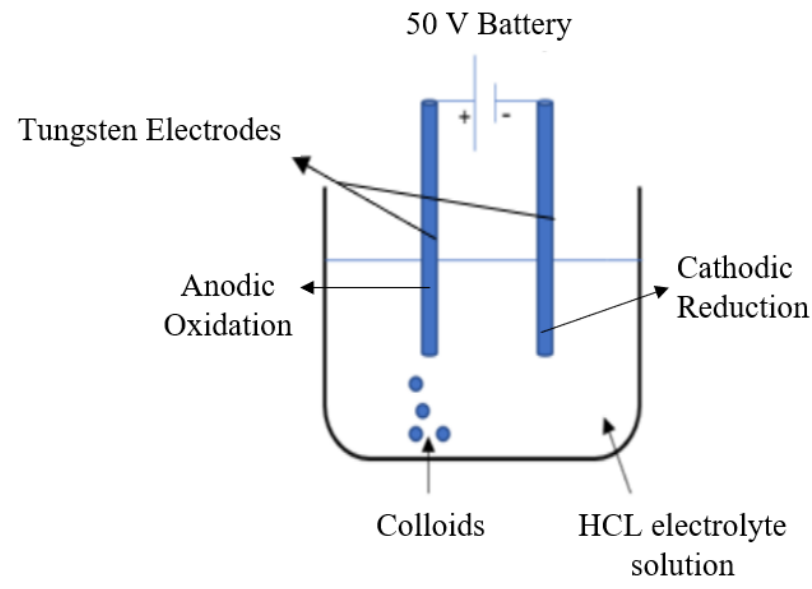

Fig. 5. Experimental setup of anodization technique.

Table 2. Advantages and disadvantages of electrochemical deposition and anodization methods.

\begin{tabular}{|c|c|c|c|c|c|}
\hline $\begin{array}{l}\text { Synthesis } \\
\text { Method }\end{array}$ & Advantages & Disadvantages & $\begin{array}{l}\text { Experimental } \\
\text { Parameters }\end{array}$ & $\begin{array}{l}\text { Particle } \\
\text { Size } \\
(\text { nm) }\end{array}$ & References \\
\hline 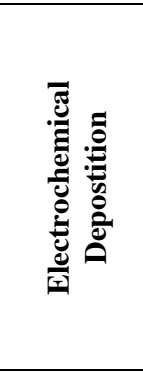 & $\begin{array}{ll}\text { - } & \text { Low capital } \\
\text { costs } \\
\text { - } \\
\text { Control of film } \\
\text { thickness by } \\
\text { changing } \\
\text { potential and } \\
\text { time }\end{array}$ & $\begin{array}{l}\text { Difficult to } \\
\text { ensure the } \\
\text { uniformity of } \\
\text { distribution of } \\
\text { particles on } \\
\text { the film }\end{array}$ & $\begin{array}{l}\text { 3.0 g of tungsten } \\
\text { metal powder was } \\
\text { dissolved in } 30 \mathrm{ml} \\
\text { of hydrogen } \\
\text { peroxide } \\
\text { Thin films were } \\
\text { controlled by } \\
\text { annealing at } 250 \\
{ }^{\circ} \mathrm{C}, 500^{\circ} \mathrm{C}, \text { and } \\
700{ }^{\circ} \mathrm{C} \\
\end{array}$ & $132-216$ & {$[19,24-25]$} \\
\hline 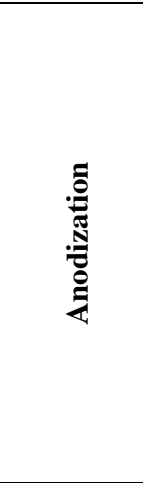 & $\begin{array}{ll}\text { - } & \text { Low } \\
& \text { processing cost } \\
\text { - } & \text { Less time } \\
& \text { consuming } \\
\text { - } & \text { Lower } \\
& \text { processing } \\
& \text { temperature } \\
\text { - } & \text { Greater control } \\
& \text { of processing } \\
\text { parameters } \\
\text { - } & \text { Stable and } \\
\text { uniform } \\
\text { structures }\end{array}$ & $\begin{array}{ll}- & \text { Lower } \\
\text { thermal } \\
\text { conducti-vity } \\
\text { of anodized } \\
\text { films }\end{array}$ & $\begin{array}{l}\text { Two tungsten rods } \\
\text { were placed } 1 \mathrm{~cm} \\
\text { parallel to each } \\
\text { other and } \\
\text { immersed into a } \\
0.02 \mathrm{M} \mathrm{HCl} \\
\text { electrolyte } \\
\text { Applied Voltage of } \\
60 \mathrm{~V}, \text { for } 5 \text { minutes } \\
\text { at } 25^{\circ} \mathrm{C}\end{array}$ & 5.1 & {$[18,26-28]$} \\
\hline
\end{tabular}

Among the various methods discussed, electrochemical oxidation or anodization process is the simplest, cheapest and effective method which is used to synthesize not only the tungsten oxide nano compounds, as well as other metal oxides such as aluminum and magnesium [31-32]. Apart from the advantage of achieving the product in relatively lower cost, nanostructures of Tungsten oxide can be achieved in low temperatures. More importantly, this study does not focus on the anodic surface, but instead on the materials 
which are released into the electrolyte medium from corrosive oxidation of the anode because nano colloids of Tungsten oxide dihydrate are released into the electrolyte medium thus a colloidal solution is obtained. Since anodization method allows the greater control on the structure formation by fine-tuning the processing parameters, it is much easier, cheaper and convenient to produce colloidal solution via any other method.

As mentioned earlier, the processing parameters of anodization have drastic effects on the formation of nanostructures thus the effects of varying the voltage, duration, electrolyte solution and temperatures, which constitute the anodization process, on the nanostructures and properties of $\mathrm{WO}_{3} \cdot 2 \mathrm{H}_{2} \mathrm{O}$ will be discussed in the following sub-section.

\subsection{Anodizing processing parameters affecting the growth of tungsten oxide nanostructures}

According to the studied journals, processing parameters used for anodization can be controlled to obtain the required properties such as uniformity in the pores of the oxide layer formed, particle size, conductivity, and antibacterial properties. Considering this, Table 3 is formed to represent the experimental parameters used by the researchers and the corresponding particle size achieved of the nano tungsten oxide structures from the studied reference journals. Therefore, the following section discusses the effects of varying processing parameters on the formation of tungsten oxide nanostructures.

Table 3. Experimental parameters used to form tungsten oxide nanostructures.

\begin{tabular}{|c|c|c|c|c|c|}
\hline \multicolumn{4}{|c|}{ Experimental Parameters } & \multirow{2}{*}{$\begin{array}{l}\text { Findings } \\
\begin{array}{c}\text { Particle Size } \\
(\mathbf{n m})\end{array}\end{array}$} & \multirow[b]{2}{*}{ Reference } \\
\hline $\begin{array}{l}\text { Electrolyte } \\
\text { Composition }\end{array}$ & $\begin{array}{c}\text { Applied } \\
\text { Voltage } \\
\text { (V) }\end{array}$ & $\begin{array}{l}\text { Temperature } \\
\left({ }^{\circ} \mathbf{C}\right)\end{array}$ & $\begin{array}{l}\text { Time } \\
(\text { min) }\end{array}$ & & \\
\hline $\begin{array}{c}\text { Aqueous electrolyte } \\
\text { containing } 1 \mathrm{M} \\
\text { ammonium sulfate and } \\
0-75 \mathrm{mM} \text { ammonium } \\
\text { fluoride }\end{array}$ & $20-50$ & $10-40$ & 240 & 90 & [7] \\
\hline $1.5 \mathrm{M}$ sulphuric acid & 20 & 50 & 240 & 32 & [18] \\
\hline $\begin{array}{c}0.02 \mathrm{M} \text { hydrochloric } \\
\text { acid }\end{array}$ & 60 & 25 & 5 & 5.1 & [26] \\
\hline $\begin{array}{c}120 \mathrm{ml} 1 \mathrm{M} \text { sulphuric } \\
\text { acid and } \\
0.5 \text { wt. \% sodium } \\
\text { fluoride }\end{array}$ & 50 & 25 & 60 & $40-50$ & [28] \\
\hline $\begin{array}{l}\text { Ethylene Glycol (EG) } \\
\text { aqueous solution }\end{array}$ & 40 & 25 & 240 & 50 & [29] \\
\hline $\begin{array}{c}0.2 \text { wt. } \% \mathrm{NH}_{4} \mathrm{~F} \\
\text { acid treatment- } \mathrm{H}_{2} \mathrm{SO}_{4}\end{array}$ & 60 & 25 & 138 & $10-15$ & [30] \\
\hline
\end{tabular}

\subsubsection{Effect of electrolyte solution}

The electrolyte solution is an important parameter which plays a significant role in the anodization process as it affects the electrochemical reactions and the oxide layer being produced [7]. Considering this, numerous journals were read to identify the effect on the 
properties and to deduce the most appropriate electrolyte solution. The electrolyte compositions from the studied journals are listed in Table 3. The table shows that the researchers have used a variety of acid compositions to perform anodization process over water or other substance. In comparison to water, acids have higher conductivity which can be suitable for synthesizing nano tungsten oxide dihydrate colloids with greater conductivity [33]. Colloids with greater electrical conductivity allow an electric current to move with little resistance which is a substantial feature for electrical conductors. Therefore, achieving the nano tungsten oxide dihydrate colloids with higher conductivity will expand potential applications of the material.

Ranjbar et al. [26] used diluted 0.02 M Hydrochloric Acid to synthesize nano-tungsten oxide dihydrate colloids via anodization method consequently, achieving colloids with the size of $5.1 \mathrm{~nm}$. Fernández et al. [18] synthesized tungsten oxide nanoplatelets using concentrated 1.5 M Sulphuric Acid for anodization process up to 240 minutes duration, resulting in $32 \mathrm{~nm}$ particle size. It was deduced that higher concentration of acids can lead to achieving the colloids of greater size whereas lower size of colloids can be achieved if dilute acids are used. Use of acids for the synthesis is also supported by a study which concludes that the dissolution of tungsten oxide takes place in acidic medium which leads to the precipitation of primary particle nuclei due to supersaturation condition. This results in the formation of inorganic fullerene-like nano tungsten oxide dihydrate colloid structure according to the series of electrochemical reactions via anodization as below [18]:

$$
\begin{aligned}
\mathrm{W}+2 \mathrm{H}_{2} \mathrm{O} & \Leftrightarrow \mathrm{WO}_{2}{ }^{2+}+4 \mathrm{H}^{+}+6 \mathrm{e}^{-} \\
\mathrm{WO}^{3}+2 \mathrm{H} & \Leftrightarrow \mathrm{WO}_{2}{ }^{2+}+2 \mathrm{H}_{2} \mathrm{O} \\
\mathrm{WO}_{2}{ }^{2+}+3 \mathrm{H}_{2} \mathrm{O} & \Leftrightarrow \mathrm{WO}_{3} \cdot 2 \mathrm{H}_{2} \mathrm{O}+2 \mathrm{H}^{+}
\end{aligned}
$$

\subsubsection{Effect of applied voltage}

Applied voltage has the significant effect on the morphology of the synthesized colloids as well as the thickness of the anodic layer. Hahn et al. [34] recommended that "harsh" anodization conditions must be established during the experiment which can lead to the breakdown events for the formation of nanostructures. Their study also discussed that applied potential is one of the most influential factor to cause the localized breakdown of anodized metals and suggested to apply the voltage with $10 \mathrm{~V}$ difference specially if the chloride containing electrolyte is used [34].

Syrek et al. [7] optimized this parameter at first during the study. For the synthesis, tungsten foil was anodized using aqueous solution of $1 \mathrm{M}$ ammonium sulfate and $0-$ $75 \mathrm{mM}$ ammonium fluoride, as mentioned in Table 2. The synthesized anodic films were obtained by testing at different voltages ranging from $20 \mathrm{~V}$ to $60 \mathrm{~V}$ with constant anodizing duration of 4 hours and $20^{\circ} \mathrm{C}$. Results from their study are plotted in Figure 6 which shows the graph of particle size against applied voltage. Their investigation through SEM images showed that films carried out at $20 \mathrm{~V}$ had the pore size of $30 \mathrm{~nm}$ with nanostructure having scattered pores, whereas the anodization carried out at $50 \mathrm{~V}$ resulted in uniform pores with $90 \mathrm{~nm}$ particle size. Thus, a gradual increase in particle size was observed in the graph with the increase in applied voltage. In contrast, applying the potential of $60 \mathrm{~V}$ or higher resulted in much thinner layer, formed with "clogged pore mouth" structure [8].

Similarly, Yang et al. [14] studied the effect of voltage on nanoporous tungsten oxide film structure by anodic oxidation. Sputtered Tungsten layer was used in a typical anodization setup with graphite sheet as counter electrode. The experiment was conducted 
at variable voltages, i.e. $20 \mathrm{~V}, 30 \mathrm{~V}, 50 \mathrm{~V}$ and $80 \mathrm{~V}$ for 60 minutes, respectively. The particle size obtained from the experiment can be observed from Figure 6. For $20 \mathrm{~V}$, nanoporous structure was formed with the particle size of about $25 \mathrm{~nm}$. When the voltage was increased to $30 \mathrm{~V}$, increase in the particle size was noticed as $43 \mathrm{~nm}$ pore size was obtained. The porosity of the surface increased and stable structure was obtained at $50 \mathrm{~V}$ but raising the potential to $60 \mathrm{~V}$ or $80 \mathrm{~V}$ destroyed the pore structure and the film consisted of agglomerated oxide particles thus the particle size of the obtained structure also reduced to just above $50 \mathrm{~nm}$ [14]. Therefore, both the studies concluded that a gradual increase in particle size was witnessed with increase in applied voltage, but the thickness of oxide layer and nanostructure morphology is compromised at applied potential of above $50 \mathrm{~V}$. It can be deduced from this conclusion that the potential of $50 \mathrm{~V}$ is most suitable for the synthesis of tungsten oxide material as regular and stable nanostructures are achieved.

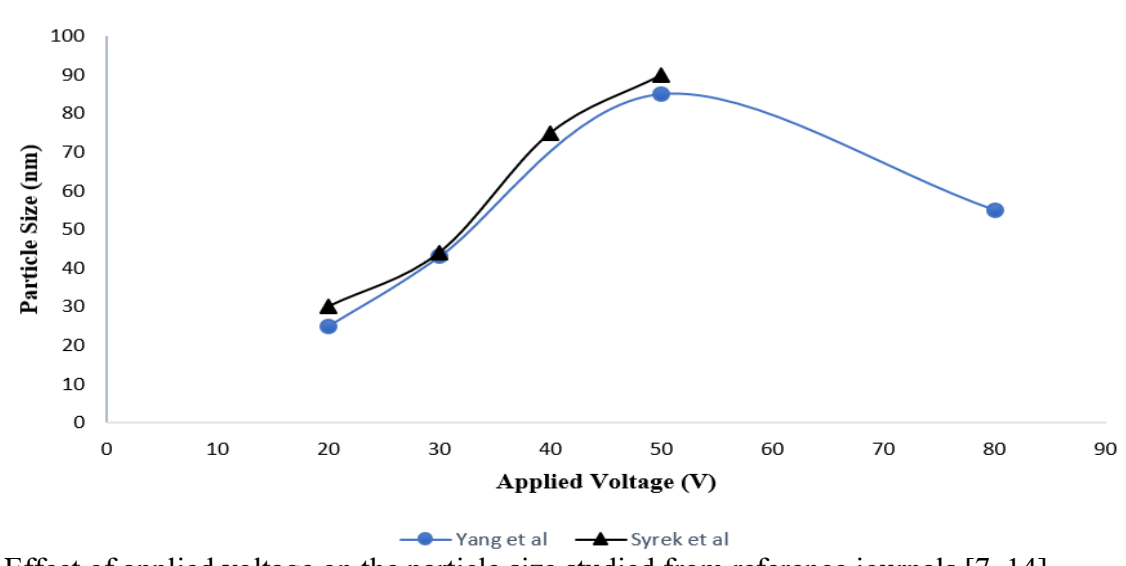

Fig. 6. Effect of applied voltage on the particle size studied from reference journals [7, 14].

\subsubsection{Effect of anodizing duration}

Time is another major factor involved in anodization which has a major impact on the properties of synthesized material. Tacconi et al. [35] being the first one to examine the effect of constant voltage on $\mathrm{WO}_{3}$, reported in his journal that at low anodization time of 30 minutes, the nanostructure of tungsten oxide was not fully developed whereas if the anodization was carried out at constant voltage for over 4 to 6 hours, pore architectures were disrupted and a disordered appearance of the colloids was observed therefore he was found to have well developed structure between 1 and 3 hours of anodization. In other studies, Syrek et al. [7] performed anodization at $50 \mathrm{~V}$ with temperatures ranging from 15 to 360 minutes. In their investigation, it was found that duration of 15 to 30 minutes initiated the anodic layer formation, but a well-defined porous morphology was only observed between 180 and 240 minutes of duration.

Charlene et al. [28] monitored the growth of tungsten oxide structures using SEM images when anodization was conducted in the presence of fluoride ions with applied voltage of $50 \mathrm{~V}$, with variable duration from 10 to 60 minutes. Figure 7 shows the results obtained at specified durations with constant potential. According to their findings, nanostructures appeared with disordered pits after 10 minutes of anodization while increasing the time to 20 minutes developed the porous structure of the oxide with the particle size of approximately $60 \mathrm{~nm}$. He also examined that $75 \mathrm{~nm}$ diameter of pore size was achieved when tungsten foil was anodized for 40 minutes and flower-like structure was also noticed. These flower-like structures only started to form after anodization of 40 
minutes which shows that these structures require soluble species which are developed by the initial anodic growth of the oxide layer. By continuing the experiment to 60 minutes of anodizing, thinner oxide layer was observed as the particle size reduced to $42 \mathrm{~nm}$ [28].

On the other hand, Yang et al. [14] characterized tungsten oxide films for different durations of 30, 45, 90 and 120 minutes by keeping the constant anodizing potential of $30 \mathrm{~V}$. The results show that insignificant morphological changes were observed but the particle size increased to $53 \mathrm{~nm}$ when anodizing duration was increased to 60 minutes but in contrast, increasing the duration above 60 minutes reduces the particle size drastically as the particle size of $45 \mathrm{~nm}$ was achieved with prolonged anodization of 120 minutes [14]. This indicates that pores were formed and enlarged at 60 minutes of anodization process.

The results of both the curves are found to be similar as the particle size increases initially with regular nanostructure properties but the prolonged duration causes the decrease in particle size. The chemical explanation for the decrease in particle size after specific duration is that the dissolution rate decreases but at the same time, oxide growth rate increases at a low anodizing voltage with prolonged duration [14]. It can be concluded from both the studies that lesser anodizing duration will result in smaller particles but ideal range to characterize the tungsten oxide nanostructures is from 20 to 60 minutes considering that particle size increases with stability and even porosity in the nanostructures.

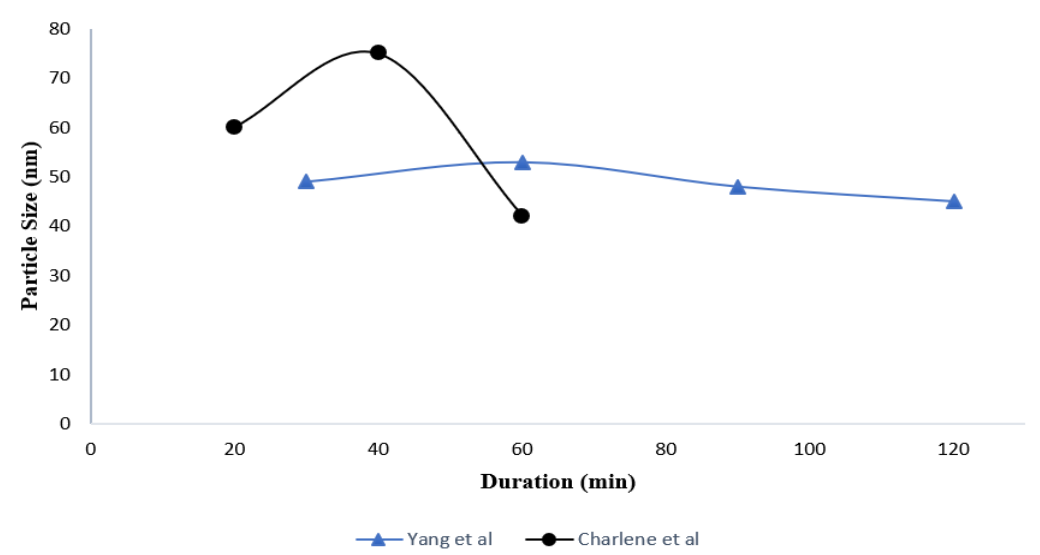

Fig. 7. Effect of anodizing duration on the particle size studied from reference journals [14, 28]

\subsubsection{Effect of anodizing temperature}

The temperature at which anodization is conducted at, has a vital importance as stated earlier in the paper but considering the data in Table 2, only few researchers have performed anodization by varying temperature while applying constant voltage. It can be observed from the table that majority of the researchers have performed the anodization at room temperature [28, 30]. Syrek et al. [7] was the first researchers investigated the effect of anodizing temperature on the nanostructures of tungsten oxides. The study anodized tungsten oxide foil from temperature range of 10 to $40{ }^{\circ} \mathrm{C}$ for 240 minutes at constant $60 \mathrm{~V}$. In their findings, elevated temperature allowed faster initiation of the formation of the nanostructures, but it also caused the metal to form anodic layer with irregular and clogged pores at times. The study also concluded that increasing the anodization temperature leads to the development of much thinner anodic films. The reason was linked to the phenomenon that the rate of chemical dissolution of oxide is increased more by raising the 
temperature than by the rate of oxide growth. Summarizing, the oxide dissolution is a predominant process at higher temperatures that results in the formation of thinner oxide layers thus affecting the size of overall structure. This study also recommends the future researchers to perform anodization at room temperature for the synthesis of nano tungsten oxide compounds, which results in stable structures and thick oxide layers of the synthesized material.

\section{Conclusion and Recommendation}

The primary objective of this paper was to review the multiple methods which are being used by the researchers in past ten years to synthesize nano tungsten oxide and recommend the most suitable method for its synthesis. The research primarily focused on Sol-gel synthesis, hydrothermal synthesis, electrochemical deposition and anodization. The impacts of each of the methods on the particle size of synthesized material are reviewed. Particularly, anodization was found to be the easiest, efficient and cost-effective method to synthesize the colloidal solution of nano tungsten oxide dihydrate which is the reason why it has been chosen by various researchers over the recent years. More significantly, this study focused on the materials which are released into the solution rather than the deposition on the anodic surface thus Anodization is most appropriate technique deduced and is recommended for the future research work.

In addition, the effects of anodizing processing parameters such as electrolytic solution, voltage and duration on the properties of synthesized material were also studied. Conclusively, if higher conductivity is the priority, dilute Hydrochloric Acid can be used as an electrolytic solution or fluoride ion containing solutions can be preferred for higher particle size. Anodizing potential of $50 \mathrm{~V}$ with duration of 60 minutes at room temperature can achieve the tungsten oxide with particle size in the range of $40-60 \mathrm{~nm}$. This range of particle size is ideal for tungsten oxide to be used in various technological applications, particularly smart windows. Also, this set of parameters will give stable and regular nanostructures of tungsten oxide.

Based on all the studied papers, a comparative research was conducted but the minimal past literature hindered the thoroughness of the review. Considering this, research gaps were identified, and it is recommended that future works should be conducted to further improve the fabrication of tungsten oxide dihydrate so that its industrial applications can be identified.

Since most of the researchers only performed the synthesis of tungsten oxide dihydrate, followed by the evaluation of characterization performed, the detail of the appropriate application of material in the future was not found. Instead of working out the best synthesis methods, researchers should try to investigate the potential of tungsten oxide dihydrate nanocolloid since it can be obtained in significantly nano size and possesses useful properties.

There was scarcity of data on the influence of particle size on conductivity and antibacterial property of the nanocolloid. Future researchers can make use of this research gap and extend this study to improvise the synthesis methods so that the desired properties of tungsten oxide dihydrate can be achieved in the future.

Furthermore, the effect of anodizing temperature on the nanostructures is not studied in detail yet. Future researchers should consider this factor to synthesize tungsten oxide at variable temperatures with constant voltage and duration as it can have significant effect on conductivity and chromic properties as well as the stability of the structures. 
Chemical and biological pollutants like bacteria and virus are extremely hazardous to the environment as well as for the human beings. Visible light active semiconductor photocatalytic property allows the possibility of utilizing the broad spectrum of abundant natural light for the degradation of chemical and biological pollutants. Since Tungsten oxide dihydrate possesses photocatalytic properties, researchers must identify its antimicrobial potentials and utilize it find the solutions to ecological problems.

\section{References}

1. B.A. Wasmi, A.A. Al-Amiery, A.A. H. Kadhum, A.B. Mohamad, J. Nanomater. 2014, 1 (2014)

2. V.B. Kumar and D. Mohanta, Bull. Mater. Sci. 34, 435 (2011)

3. H.I.S. Nogueira, A.M.V. Cavaleiro, J. Rocha, T. Trindade, J.D.P. De Jesus, Mater. Res. Bull. 39, 683 (2004)

4. L. Ghasemi, H. Jafari, Mater. Res. 20, 1713 (2017)

5. M. Yang, N.K. Shrestha, P. Schmuki, Electrochem. commun. 11(10), 1908 (2009)

6. G. Orsini, V. Tricoli, J. Mater. Chem. 20, 6299 (2010)

7. K. Syrek, L. Zaraska, M. Zych, G.D. Sulka, J. Electroanal. Chem. 829, 106 (2018)

8. A. Yan, C. Xie, D. Zeng, S. Cai, M. Hu, Mater. Res. Bull. 45, 1541 (2010)

9. A.P. Baker, S.N.B. Hodgson, M.J. Edirisinghe, Surf. Coat. Technol. 153, 184 (2002)

10. C C. Mardare, A.W. Hassel, Phys. Status Solidi. A. 216, 1 (2019)

11. Y. Chai, F.Y. Ha, F.K. Yam, Z. Hassan, Procedia. Chem. 19, 113 (2016)

12. H. Ahmadian, F.S. Tehrani, M. Aliannezhadi, Mater. Res. Exp. 6, 105024 (2019)

13. H. Zheng, J.Z. Ou, M.S. Strano, R.B. Kaner, A. Mitchell, K. Kalantar-Zadeh, Adv. Func. Mater. 21, 2175 (2011)

14. T. Yang, Y. Zhang, Y. Cai, H. Tian, J. Mater. Res., 29, 166 (2014)

15. S.J. Wang, M.C. Wang, S. Chen, Y.H. Li, T.S. Shen, H. Bor, C.N. Wei, Sensors. 18, $2803(2018)$

16. C.M. Wu, S. Naseem, M.H. Chou, J.H. Wang, Y.Q. Jian, Front. Mater. 6, 1 (2019)

17. T. Kikuchi, J. Kawashima, S. Natsui, R.O. Suzuki, Appl. Surf. Sci. 422, 130 (2017)

18. R.M. Fernandez-Domene, R. Sanchez-Tovar, E. Segura-Sanchis, J. Garcia-Anton, Chem. Eng. J. 286, 59 (2016)

19. P.G. Jamkhande, N.W. Ghule, A.H. Bamer, M.G. Kalaskar, J. Drug Deliv. Sci. Technol. 53, 101174 (2019)

20. A.E. Danks, S. R. Hall, Z. Schnepp, Mater. Horiz. 3, 91-112 (2016)

21. M. Alsawafta, Y.M. Golestani, T. Phonemac, S. Badilescu, V. Stancovski, V.V. Truong, J. Electrochem. Soc. 161, H276 (2014)

22. H. Ahmadian, F.S. Tehrani, M. Aliannezhadi, Mater. Res. Exp. 6, 105024 (2019)

23. L. Wang, H. Hu, J. Xu, S. Zhu, A. Ding, C. Deng, J. Mater. Res. 34, 2955 (2019)

24. L. Lin, C. P. Cheng, T. P. Teng, J. Nanomater. 2016, 25 (2016)

25. A.J. More, R.S. Patil, D. S. Davali, M. P. Suryawanshi, V.V. Burungale, J.H. Kim, P. S. Patil, Mater. Lett. 134, 298 (2014)

26. M. Ranjbar, A. H. Fini, H. Kalhori, P. Kameli, H. Salamati, Sol. Energ. Mat. Sol. C. 132, 329 (2015)

27. K. Syrek, M. Zych, L. Zaraska, G. D. Sulka, Electrochim. Acta. 231, 61 (2017)

28. C. Ng, C. Ye, Y.H. Ng, R. Amal, Cryst. Growth Des. 10, 3794 (2010)

29. L. Zaraska, K. Gawlak, M. Gurgul, D.K. Chlebda, R.P. Socha, G.D. Sulka, Electrochim. Acta. 54, 238 (2017)

30. C. Wang, C. Lin, S. Wang, Electrochim. Acta, 112, 24 (2013) 
31. S. Wu, Y. Li, X. Chen, J. Liu, J. Gao, G. Li, Electrochem. Commun. 104, 106479 (2019)

32. L. Santos, J. P. Neto, A. Crespo, P. Baiao, P. Barquinha, L. Pareira, R. Martins, E. Fortunato, Electroplating of Nanostructures (Intech, Croatia, 2015)

33. H. Golnabi, M.R. Matloob, M. Bahar, M. Sharifian, Iranian Phys. J. 3, 24 (2009)

34. R. Hahn, J.M. Macak, P. Schmuki, Electrochem. commun. 9, 947 (2007)

35. N.R. De Tacconi, C.R. Chenthamarakshan, G. Yogeeswaran, A. Watcharenwong, R.S. de Zoysa, N.A. Basit, K. Rajeshwar, J. Phys. Chem. B. 110, 25347 (2006) 